MONS. ANTONIO INTERGUGLIELMI

Studium Theologicum Galilaeae, Israel

\title{
LA PREPARAZIONE DEGLI SPOSI AL MATRIMONIO: UNA PANORAMICA PASTORALE-GIURIDICA
}

Sommario: Premessa. - 1. La Preparazione al Matrimonio: lo sviluppo per adeguarla alla nuova realtà. -2 . Documenti e norme sulla preparazione al matrimonio. - 3. La preparazione alle nozze oggi: il "Catecumenato" per gli sposi suggerito da Papa Francesco. - 4. Corsi di preparazione al matrimonio e l'accompagnamento dei fidanzati: possono essere un aiuto per la immaturità che rende spesso nullo il matrimonio?

\section{Premessa}

Papa Francesco nell'ultima Allocuzione al Tribunale della Rota Romana, tenuta il 22 gennaio 2016 ha affermato: "La Chiesa, dunque, con rinnovato senso di responsabilità continua a proporre il matrimonio, nei suoi elementi essenziali - prole, bene dei coniugi, unità, indissolubilità, sacramentalità -, non come un ideale per pochi, nonostante i moderni modelli centrati sull'effimero e sul transitorio, ma come una realtà che, nella grazia di Cristo, può essere vissuta da tutti $i$ fedeli battezzati. E perciò, a maggior ragione, l'urgenza pastorale, che coinvolge tutte le strutture della Chiesa, spinge a convergere verso un comune intento ordinato alla preparazione adeguata al matrimonio, in una sorta di nuovo catecumenato - sottolineo questo: in una sorta di nuovo catecumenato - tanto auspicato da alcuni Padri Sinodali"'.

\footnotetext{
${ }^{1}$ Papa Francesco, Allocuzione alla Rota Romana del 22 gennaio 2016, L'Osservatore Romano, 23 gennaio 2016.
} 
Queste parole del Papa, indirizzate al massimo organo di controllo giurisdizionale della Chiesa Cattolica, fanno emergere una prospettiva interessante, anche alla luce della recente riforma del processo per la dichiarazione di nullità matrimoniale: la necessità, cioè, di un virtuoso collegamento tra la prassi pastorale e quella giudiziaria, ai fini di una "adeguata preparazione" dei fidanzati al matrimonio.

In queste riflessioni ci interessa dunque approfondire come la questione dello sviluppo dei corsi di preparazione al matrimonio, previsti dal Codice al can. 1063, possa trarre dalla prassi dei Tribunali Ecclesiastici delle indicazioni utili per fare dei passi avanti nella pastorale di accompagnamento dei fidanzati (e degli sposi) ${ }^{2}$.

L'idea è che questo sviluppo, reso necessario dalle crescenti difficoltà che incontrano i matrimoni, possa trovare proprio dalla realtà giudiziaria delle preziose indicazioni, poiché la prassi giurisdizionale delle cause di nullità matrimoniali mette in luce realtà di fallimento spesso già annunciate, ma non riconosciute o aiutate in modo adeguato ${ }^{3}$.

\section{La Preparazione al Matrimonio: lo sviluppo per adeguarla alla nuova realtà}

L'argomento dei corsi di preparazione al matrimonio, già oggetto di approfonditi e pregevoli lavori e di alcune indicazioni da parte del Dicastero della Famiglia, trova oggi nuovi spunti di sviluppo alla luce proprio delle possibili prospettive aperte dalla ricordata Allocuzione

\footnotetext{
${ }^{2}$ In questo senso già alcuni studi negli anni scorsi, tra cui: T. PocAlujko, $L a$ prevenzione della nullità del matrimonio nella preparazione e nell'ammissione alle nozze con una considerazione del contributo dei tribunali ecclesiastici, Ed. PUG Serie Diritto Canonico nr. 89, Roma, 2011. Alcuni spunti anche in G. CaberLetTi, La collaborazione tra pastori d'anime e tribunali ecclesiastici in relazione alle cause di nullità matrimoniale, Quaderni di Diritto Ecclesiale 21(2007), pp. 415-435.

${ }^{3}$ Cfr. sull'argomento, con un profilo rivolto prevalentemente ai corsi di preparazione al sacramento, P. BIANCHI, Nullità del matrimonio e difetti nella sua preparazione, Quaderni di diritto Ecclesiale 1(1988) e L. GHIsoni, La prevenzione della nulità del matrimonio nella preparazione immediata alle nozze, Periodica 100(2015), pp. 477-501.
} 
di Papa Francesco e dai lavori della III Assemblea Straordinaria del Sinodo, dedicata alla Famiglia.

In diverse Diocesi del mondo già si stanno sviluppando iniziative per rendere più adeguata alla situazione reale la pastorale famigliare, intendendo con questa espressione in primo luogo l'accompagnamento dei fidanzati al matrimonio. Interessante che, ad esempio, in un'approfondita indagine sui corsi di preparazione al matrimonio, svolta diversi anni fa per conto della Conferenza Episcopale degli Stati Uniti ${ }^{4}$, già si rilevavano alcune carenze da colmare, necessità oggi divenuta non più rinviabile.

Nelle conclusioni di questa ricerca, che risale all'anno 1995, infatti si rilevava che, nell'elaborare i programmi dei corsi di preparazione al matrimonio, era necessario comprendere meglio le dinamiche e le necessità delle coppie all'interno della Chiesa: "The challenge is clear. Those who provide marriage preparation programs need to understand better the dynamics and needs of inter-church couples so that they can respond to them better and offer them programs more suited to the demands of their situations".

In questa prospettiva, non c'è dubbio che le cause di richiesta di accertamento della nullità del matrimonio sono lo specchio dei notevoli cambiamenti che si sono verificati in questi anni nella comunità sociale ed ecclesiale ${ }^{6}$ e quindi della necessità di un adeguamento della pastorale.

\footnotetext{
${ }^{4}$ Cfr. Study of the Value of Marriage Preparation in the Catholic Church for Couples Married One Through Eight Years, Center for Marriage and Family Creighton University, novembre 1995, in http://www.usccb.org/ issues-and-action/marriage-and-family/marriage/marriage-preparation/ getting-it-right-marriage-preparation-in-the-catholic-church.

${ }^{5}$ Cfr. op.ult.cit.

${ }^{6}$ In un recente studio dedicato alla partecipazione dei laici alla preparazione del matrimonio canonico si afferma; "La famiglia si trova ad affrontare l'impresa di far valere la sua vera identità: un'unione tra due persone eterosessuali, fondata sul matrimonio ed ordinata al bene dei coniugi e dei figli'. Così in Veronica D’Alleva, La partecipazione dei laici nella preparazione giuridico-pastorale al matrimonio canonico, Città del Vaticano, 2010, p. 111. Sullo stesso argomento, cfr. anche AA.VV.,
} 
Si tratta non solo del processo di scristianizzazione già in atto da decenni soprattutto nei paesi occidentali, ma anche di diversi cambiamenti sociali: si pensi, ad esempio, al mutamento del ruolo della donna nella società e quindi, di riflesso, nella famiglia, oppure alle nuove recenti sfide che il matrimonio è chiamato ad affrontare soprattutto in molti Paesi che, oramai anche da un punto di vista normativo, dimostrano di non favorire affatto - talvolta anzi di voler indebolire - la famiglia creaturale, così come intesa dalla dottrina cristiana.

Inutile sottolineare di come si tratti di un'analisi molto complessa perché, partendo da dati concreti, richiederebbe di svilupparsi con alcune articolazioni geografiche: molti dei problemi all'origine dei fallimenti dei matrimoni nelle società Occidentali non sempre sono gli stessi dell'America Latina o dell'Africa ${ }^{7}$.

Del resto, anche nella Relazione finale del Sinodo dei Vescovi a Papa Francesco, del 24 ottobre 2015, viene ribadito questo dato, da cui non si può più prescindere: "Alcuni cattolici hanno difficoltà a condurre le loro vite in accordo con l'insegnamento della Chiesa cattolica sul matrimonio e la famiglia, e a vedere in tale insegnamento la bontà del progetto creativo di Dio per loro. I matrimoni in alcune parti del mondo diminuiscono, mentre le separazioni e i divorzi non sono rari".

Da qui il criterio unificatore che emerge non solo nella pastorale ma anche dalla pratica giudiziaria: la comune esigenza di una sempre più accurata preparazione dei futuri coniugi, che divenga una sorta di "nuovo catecumenato", come ha affermato a gennaio scorso Papa

Ammissione alle nozze e prevenzione della nullità del matrimonio, a cura di M.A. Ortiz, Milano, 2005.

${ }^{7}$ Agapit J. Mroso, The Church in Africa and the New Evangelisation, Roma, 1995, ed. PUG - Serie teologica 6, a p. 143 scrive in proposito "If there is one thing that the Bishop of Africa were unanimous on during the 1980 Synod it was the need for marriage preparation to incorporate the values of African family life. In light of serious contemporaney problems facing Christian marriages, preparation is absolutely needed." 
Francesco nella ricordata Allocuzione alla Rota, riprendendo le indicazioni del Sinodo sulla Famiglia ${ }^{8}$.

\section{Documenti e norme sulla preparazione al matrimonio}

Il canone 1063 sulla preparazione al matrimonio accoglie la dottrina del Vaticano II, in particolare il numero 52 della Costituzione conciliare Gaudium et Spes ${ }^{9}$.

Preziose indicazioni sui compiti dei pastori nella preparazione al matrimonio si trovano anche nei documenti del precedente Sinodo dei Vescovi del 1980 dedicato alla famiglia, tenutosi a Roma dal 26 settembre al 25 ottobre 1980: tra l'altro veniva ribadito il compito delle Conferenze Episcopali e delle Diocesi di pubblicare "Direttori pastorali" per la famiglia, in attuazione del canone 1064 e 1067, nei quali dare ai pastori le indicazioni per la preparazione el'accompagnamento delle famiglie.

Questi "Direttori” devono contenere anche le disposizioni sulle modalità da seguire per organizzare e strutturare i corsi di preparazione al matrimonio: in specie, viene sottolineata la necessità di porre al centro dei medesimi l'aspetto della formazione alla vita di fede, e non solo (comunque non principalmente) quello intellettuale o nozionistico ${ }^{10}$.

\footnotetext{
${ }^{8}$ Papa Francesco, Allocuzione alla Rota Romana del 26 gennaio 2016, cit.

${ }^{9}$ Concilio Vaticano II, Cost. past. Gaudium et spes, 22, AAS 58(1966), dove si legge tra l'altro: "É compito dei sacerdoti, provvedendosi una necessaria competenza sui problemi della vita familiare, aiutare amorosamente la vocazione dei coniugi nella loro vita coniugale e familiare con i vari mezzi della pastorale, con la predicazione della parola di Dio, con il culto liturgico o altri aiuti spirituali, fortificarli con bontà e pazienza nelle loro difficoltà e confortarli con carità, perché si formino famiglie veramente serene. Le varie opere di apostolato, specialmente i movimenti familiari, si adopereranno a sostenere con la dottrina e con l'azione i giovani e gli stessi sposi, particolarmente le nuove famiglie, ed a formarli alla vita familiare, sociale ed apostolica".

${ }^{10}$ Cfr. Assemblea Generale del Sinodo dei Vescovi, De familiae christianae muneribus in mundo huius temporis, 26.09.1990-25.10.1990, in Enchiridion Vaticanum, vol. VII, 1982, nn. 805-806, pp. 739-741.
} 
Altre indicazioni fondamentali per i pastori e per tutti coloro che nella comunità Ecclesiale si dedicano al servizio della pastorale famigliare $^{11}$ si trovano nell'Esortazione apostolica Familiaris consortio di Papa Giovanni Paolo II del 1981, che raccoglie appunto le conclusioni del ricordato Sinodo del $1980^{12}$.

Più recentemente, il 13 maggio 1996, il Pontificio Consiglio per la Famiglia ha pubblicato altre indicazioni nel documento La Preparazione al Matrimonio ${ }^{13}$, in cui si legge tra l'altro: "Ci sono atteggiamenti e misure, contemplate nelle legislazioni, che non aiutano la famiglia fondata sul matrimonio e negano perfino i suoi diritti. Difatti, un'atmosfera di secolarizzazione si è andata diffondendo in diverse parti del mondo e coinvolge specialmente i giovani e li sottomette alla pressione di un ambiente di secolarismo nel quale si finisce per perdere il senso di Dio e di conseguenza si perde anche il senso profondo dell'amore sponsale e della famiglia"14.

Una constatazione oggi più che attuale, di fronte alla quale dobbiamo adeguare il nostro intervento a favore della costruzione (o ri-costruzione) della famiglia cristiana.

\section{La preparazione alle nozze oggi: il "Catecumenato" per gli sposi suggerito da Papa Francesco}

Non c'è dubbio che il progressivo generalizzato processo di allontanamento dalla fede cristiana in molti Paesi Occidentali ha ricevuto

\footnotetext{
${ }^{11}$ Giovanni Paolo II nell'Omelia della Messa celebrata il 25 ottobre 1980 a Conclusione della V Assemblea Generale del Sinodo dei Vescovi ribadiva la cura della famiglia come un compito di tutta la comunità ecclesiale: "Abbiamo constatato, quanto amore di Cristo, quanta carità è offerta a tutti coloro che nella Chiesa e nel mondo formano una famiglia: non solo agli uomini e alle donne riuniti in matrimonio, ma anche ai ragazzi e ragazze, ai giovani, ai vedovi, e agli orfani, agli anziani e a tutti quelli che in qualche modo partecipano alla vita della famiglia".

${ }^{12}$ Giovanni Paolo II, Esortazione apostolica Familiaris consortio, 22 novembre 1981, AAS 74(1982).

${ }^{13}$ Pontificio Consigilio per la Famiglia, Preparazione al Sacramento del matrimonio, in Enchiridion Vaticanum, vol. XV, 1996.

${ }^{14}$ Pontificio Consigilio per la Famiglia, cit., nr 11.
} 
un impulso determinante dalla rivoluzione socio-culturale della fine degli anni '60 e degli anni '70: le generazioni successive in qualche modo sono il risultato di famiglie che spesso hanno perso la Chiesa come punto di riferimento, cercando altrove la loro identità. I dati di progressiva diminuzione percentuale dei bambini battezzati anche in Italia indicano chiaramente che non è più sentito il dovere, di cui anche al canone $774 \$ 2$ C.I.C., di educare cristianamente i figli ${ }^{15}$.

La debolezza nel rapporto con la fede cristiana fa sì che le ultime generazioni di fidanzati che decidono di celebrare in chiesa la loro unione abbiano molte difficoltà nel concepire la portata e il valore del matrimonio cristiano, soprattutto per quanto riguarda i risvolti concreti connessi all'indissolubilità del vincolo, all'apertura alla trasmissione del dono della vita e alla fedeltà.

Si tratta di temi che qualsiasi corso di preparazione al matrimonio tratta, più o meno approfonditamente. Il punto debole è che spesso sono affrontati come se fossero già acquisiti dai fidanzati, mentre si tratta di tematiche che risultano nuove e di difficile comprensione: un linguaggio quasi incomprensibile, per chi è rimasto spesso a qualche nozione elementare del catechismo della prima comunione o della cresima.

E’ questione dunque non solo di adeguare il linguaggio, ma anche la forma, i tempi, le modalità ed il contenuto di questo accompagnamento alla realtà della fede (non) vissuta: bisogna attivare dinamiche che superino non solo la mancanza di conoscenza e di formazione catechetica, ma anche l'insensibilità/indifferenza di fondo verso la realtà ecclesiale, su cui il matrimonio dovrebbe invece fondare meglio la propria identità, in una connessione vitale tra la prima comunità umana e quella cristiana.

\footnotetext{
${ }^{15}$ Così anche Familiaris Consortio n. 36: "Il diritto dovere dei genitori si qualifica come essenziale, connesso com'è con la trasmissione della vita umana..”, e anche al numero 38 Giovanni Paolo II afferma: "tale è la grandezza e lo splendore del ministero educativo dei genitori cristiani, che san Tommaso non esita a paragonarlo al ministero dei sacerdoti..”, Giovanni PaOlo II, Esortazione apostolica Familiaris consortio, cit.
} 
La più attenta e approfondita preparazione al matrimonio che si dovrebbe realizzare progressivamente in molte Diocesi e Parrocchie, potrebbe essere particolarmente efficace per evitare casi di nullità frequenti, come quelli connessi all'errore circa le proprietà essenziali del matrimonio ${ }^{16}$ (che peraltro, come noto e come confermato dalla giurisprudenza dei Tribunali Ecclesiastici, vizia il consenso solo se il soggetto esclude positivamente il consenso circa una delle proprietà essenziali o la dignità del sacramento) ${ }^{17}$.

Più complesso invece appare il caso di incapacità di assumere gli oneri (can. $1095 \$ 2$ C.I.C.) ${ }^{18}$.

La giurisprudenza tende alla valorizzazione sempre maggiore dell'incapacità ad assumere gli obblighi essenziali del matrimonio, nella quale confluiscono i casi nei quali la nullità è originata dalla incapacità di determinarsi responsabilmente ad un atto così impegnativo e carico di conseguenze come è il sacramento del matrimonio.

Per evitare che si incrementi ulteriormente la crescente presentazione di cause di nullità del matrimonio per vizi legati alla mancanza di maturità personale, di cui al can. $1095 \$ 2$ C.I.C., se non è certo sufficiente arricchire i corsi con un maggior spessore teologico, pare che nemmeno un prolungamento dei tempi di preparazione e di discernimento possa essere di per sé risolutivo; né sembra sufficiente adottare tecniche di accompagnamento psicologico sempre più raffinate.

L'immaturità è spesso non facilmente valutabile come tale prima delle nozze, perlomeno da chi non ha preparazione molto specifica ed esperienza in materia; e di solito esplode con l'assunzione reale

\footnotetext{
${ }^{16}$ Ai tre bona matrimonialia di tradizione Agostiniana (bonum prolis, bonum fidei, bonum sacramenti), alcuni giudici della Rota Romana aggiungono un quarto, bonum coniugum; ma in dottrina ci sono diverse posizioni in tal senso. Ad esempio, rimane fedele alla trilogia, C. Scicluna, The essential definiotion of marriage according to the 1917 and 1983 codes of Canon Law: an exegetical and comparative study, New York, 1995, pp. 303-304.

${ }^{17} \mathrm{Cfr}$. sul punto J. KowAL, L'errore circa le proprietà essenziali o la dignità sacramentale del matrimonio (c. 1099), Periodica 87(1998), pp. 290-304.

${ }^{18} \mathrm{Ne}$ tratta tra gli altri in maniera approfondita I. GRAmUnt, Canons and commentaries on marriage, Collegeville (MN), 1987.
} 
degli oneri matrimoniali e delle conseguenti responsabilità. Per questa ragione anche nella Dignitas Connubii, l'Istruzione da osservarsi nei tribunali diocesani e interdiocesani nella trattazione delle cause di nullità del matrimonio pubblicata dal Pontificio Consiglio per i testi legislativi il 25 gennaio 2005, troviamo in diversi articoli regolamentata la prova delle lacune nella personalità dei nubendi attraverso l'adozione di perizie psicologiche di specialisti ${ }^{19}$.

Come suggerisce la pratica dei Tribunali Ecclesiastici che si occupano di nullità matrimoniale, tante volte la radice ultima delle problematiche che vengono alla luce solo dopo la celebrazione del sacramento nuziale è da ricercare non solo in un'immaturità occulta e remota esplosa improvvisamente, ma anche nel mancato accompagnamento ecclesiale e nella solitudine in cui vengono lasciati di solito i neo-coniugi dopo la celebrazione delle nozze.

La mancanza di maturità psico-affettiva dei fidanzati che contraggono un impegno serio e profondo come il matrimonio cristiano nasce prima di tutto dalla mancanza di consapevolezza del soggetto immaturo ${ }^{20}$, che contrae spesso il matrimonio in buona fede: solo messo di fronte alla quotidianità della vita comune, che chiama gli sposi a crescere in un cammino di donazione e di sacrificio comune, ci si rende conto di non aver compreso né quello che si andava ad iniziare, né tanto meno la propria realtà personale, per cui ci si ritrova privi di equipaggiamento.

Ci chiediamo allora come i sacerdoti e i gruppi di pastorale familiare possano aiutare i fidanzati e dare un valido contributo per

\footnotetext{
${ }^{19} \mathrm{Su}$ questo aspetto, cfr. M.J. Arroba CONDE, La prova peritale e le problematiche processualistiche, in AA. VV., L'incapacità di intendere e di volere nel diritto matrimoniale canonico (can. 1095 nn. 1-2), Città del Vaticano, 2000, pp. 395-396; J. Llobell, El valor jurídico de la instr. "Dignitas connubii», su recepción eclesial, el objeto y la conformidad de la sentencia, y la certeza moral, in: Procesos de nulidad matrimonial..., pp. 236-237 e F. DANEeLs, Una introducción general a la Instrucción Dignitas Connubii, Ius Canonicum 46(2006), p. 34 ss.

${ }^{20}$ E' noto come la communion personarum è composta da un elemento fisico, i due sposi, e da uno metafisico: così Gramunt, Canons..”, cit., pp. 375-376.
} 
arginare in qualche modo il moltiplicarsi delle cause di richiesta di nullità matrimoniale davanti ai Tribunali ecclesiastici.

In una cornice più ampia, merita attenzione e discernimento particolari da parte dei pastori ${ }^{21}$ anche la problematica dei matrimoni interreligiosi: come emerge da qualche anno dalle cause di nullità del matrimonio, si tratta di unioni che richiedono fin dalla fase del fidanzamento un ancor più attento accompagnamento e di un approfondito discernimento da parte dei pastori ${ }^{22}$.

\section{Corsi di preparazione al matrimonio e l'accompagnamento dei fidanzati: possono essere un aiuto per la immaturità che rende spesso nullo il matrimonio?}

I padri Sinodali rivolgendosi a coloro che esercitano il ministero pastorale a beneficio dei coniugi e delle famiglie hanno respinto ogni dicotomia tra la pedagogia, che propone una certa gradualità nel realizzare il piano divino, e la dottrina, proposta dalla Chiesa con tutte le sue conseguenze, nelle quali è racchiuso il comando di vivere secondo la stessa dottrina. Non si tratta di guardare la legge solo come un puro ideale da raggiungere in futuro, ma come un comando di Cristo Signore a superare con impegno le difficoltà.

Il Sinodo ha parlato della donna, della sua dignità e della sua vocazione come figlia di Dio, moglie e madre in modo appropriato e persuasivo, con rispetto e gratitudine. Respingendo tutto ciò che lede

\footnotetext{
${ }^{21} \mathrm{Si}$ legge ad esempio nelle conclusioni della citata indagine sui corsi di preparazione al matrimonio nella Chiesa degli Stati Uniti: "The Center for Marriage and Family is proposing a follow-up study on inter-church marriages, national and ecumenical, to gather and analyze data on them, and to create and pilot models of marriage preparation fitted to their situations", in: www.usccb. org/issues-and-action/marriage-and-family/marriage/marriage-preparation/ getting-it-right-marriage-preparation-in-the-catholic-church.

${ }^{22}$ Andrà verificata con attenzione la motivazione alle nozze della parte non cattolica: "Ciò consentirà di prevenire eventuali nullità del matrimonio per simulazione totale o per esclusione del bonum sacramenti o per condizione...” scrive L. GHIsonI, La prevenzione della nullità del matrimonio nella preparazione immediata alle nozze, op. cit., p. 485.
} 
la sua dignità umana, il Sinodo ha evidenziato anche la grandezza della dignità della madre. In virtù di questo ruolo fondamentale della donna, sposa e madre, ha dichiarato che la società deve costituirsi in modo tale che la donna non sia costretta ad un lavoro fuori casa non per una scelta libera e responsabile, ma solo per motivi economici (spesso di sussistenza): bisogna che la famiglia possa vivere convenientemente anche quando la madre si dedica totalmente ad essa.

Come indica l'Istrumentum Laboris della III Assemblea Generale Sinodo Straordinaria dei Vescovi ${ }^{23}$, troviamo risposte molto simili tra i diversi Continenti a proposito della preparazione al matrimonio: dove sia offerto l'accompagnamento di gruppi di pastorale familiare che organizzano seminari e ritiri di preghiera per coppie - che coinvolgono come animatori, oltre ai sacerdoti, anche coppie sposate di consolidata esperienza familiare - sicuramente abbiamo migliori risultati.

Sempre nel documento che ha guidato i lavori del Sinodo del 2014, si ricorda che in questi corsi si cerca di dare i fondamenti della relazione di coppia, cercando di approfondire la consapevolezza e la libertà della scelta, la conoscenza degli impegni umani e civili ma soprattutto cristiani.

Torniamo dunque ancora una volta all'indicazione di Papa Francesco: la prassi delle cause di nullità dei Tribunali Ecclesiastici ci mostra che non solo è indispensabile riprendere la catechesi dell'iniziazione cristiana alla fede, ma che la maggiore efficacia della cura pastorale si realizza dove questo accompagnamento non termina con la celebrazione delle nozze, ma 'scorta' almeno i primi anni di vita coniugale ${ }^{24}$.

\footnotetext{
${ }^{23}$ Sinodo dei Vescovi XIV Assemblea Generale Ordinaria, Instrumentum Laboris - La vocazione e la missione della famiglia nella Chiesa e nel mondo contemporaneo, Cinisello Balsamo (MI), 2015.

${ }^{24}$ Nell'Istrumentum Laboris del III Sinodo dei Vescovi, op.ult.cit., al numero 52 si trova descritta la situazione: “...Si tende per questo in molti contesti a promuovere catechesi differenziate: per i giovani anche prima del fidanzamento; per i genitori dei fidanzati; per le coppie già sposate; per le persone separate; per la preparazione al battesimo; per la conoscenza dei documenti pastorali dei Vescovi e del Magistero della Chiesa. In qualche Paese si segnalano vere e proprie scuole di preparazione alla
} 
Non è sufficiente far conoscere intellettualmente il contenuto della fede e del matrimonio ma è necessario acquisire gli strumenti e il supporto per viverlo: e questo non può che avvenire attraverso un percorso di crescita nella fede delle coppie, che non si esaurisce certo in qualche mese, con una decina di incontri.

Così anche San Giovanni Paolo II, nel suo discorso alla Rota Romana del 1999, ribadiva la necessità di una più attenta preparazione alle nozze: "Mi limito a sottolineare la grave responsabilità che incombe ai Pastori della Chiesa di Dio di curare una adeguata e seria preparazione dei nubendi al matrimonio: solo cosi, infatti, si possono suscitare nell'animo di coloro che si apprestano a celebrare le nozze le condizioni intellettuali, morali e spirituali, necessarie per realizzare la realtà naturale e sacramentale del matrimonio" 25 .

Occorre a nostro parere porsi oggi questa domanda impegnativa: stante la debolezza della fede che spesso si riscontra nelle coppie che si avvicinano al matrimonio, tale da rendere insufficienti i pochi incontri di preparazione al matrimonio, non è forse necessario considerare il sacramento del matrimonio in modo nuovo, come un percorso che va oltre la celebrazione delle nozze?

Questa esigenza trova conferma dal fatto che, quando oggi andiamo ad analizzare le decisioni rotali degli ultimi anni, troviamo ancora molte cause fondate sull'esclusione del bene della prole, dell'indissolubilità e talvolta del bonum fidei, ma in percentuale il loro numero è in diminuzione; mentre aumentano notevolmente le cause sull'incapacità psico-affettiva. Emerge dunque la necessità di un accompagnamento delle giovani coppie nel processo di maturazione, non solo di fede ma anche affettiva e relazionale. Per cui il matrimonio

vita matrimoniale, indirizzate soprattutto alla istruzione e promozione della donna. Il discorso si differenzia in particolare nelle zone in cui vi è una forte secolarizzazione, ove si constata una crescente distanza culturale delle coppie nei confronti dell'insegnamento della Chiesa".

${ }^{25}$ Giovanni Paolo II, Discorso alla Rota Romana, 21 gennaio 1997, n. 7, AAS 91(1991), p. 627. 
potrebbe venire considerato come un'occasione e una tappa di crescita e maturazione nella fede e nella maturità personale.

Si tratta allora di interpretare in modo nuovo i corsi di preparazione, di scoprire una nuova via: il desiderio dei fidanzati di celebrare un matrimonio in chiesa può divenire l'occasione per una ripresa di contatto con le sorgenti della fede, che essi stessi spesso riconoscono molto debole e influenzata dai disvalori di una società che porta avanti scelte e modelli molto o del tutto non cristiani ${ }^{26}$.

A questo punto è l'apporto della comunità ecclesiale, di cui la famiglia è cellula fondamentale, che emerge. Non solo i pastori ma anche, insieme con loro, le coppie di fedeli laici impegnate nella pastorale familiare possono divenire un aiuto e un punto di riferimento prezioso per i giovani coniugi: un'opportunità nuova per riscoprire che essi fanno parte, come famiglia cristiana, di una comunità che continua ad accompagnarli e a sostenerli anche dopo la celebrazione delle nozze.

Le esigenze pastorali che nascono dalla mutata realtà sono dunque nuove, ma identica rimane la visione ecclesiale. La conversatio coniugali, cioè la famiglia reinterpretata alla luce della Fede è sempre stata un "ministero di grazia non solo per i coniugi". Già Pio XI, riprendendo il cardinale Bellarmino, scriveva: "il matrimonio si può riguardare in due modi: il primo mentre si celebra; il secondo mentre perdura dopo che è stato celebrato... perché fin quando vivono i coniugi, la loro unione è sempre il sacramento di Cristo e della Chiesa"27.

\footnotetext{
${ }^{26}$ Si pensi al capo di nullità che riguarda l'esclusione del bonum prolis: la giurisprudenza considera l'esclusione ad tempus, per motivi transeunti, come un motivo che non rende nullo il matrimonio. Indubbiamente l'esclusione temporanea, cioè la programmazione della nascita dei figli, è diffusissima, ma questo mostra chiaramente un modo di vivere della famiglia lontano dai valori del matrimonio cristiano.

${ }^{27}$ PIO XI, AAS 22(1930), p. 583.
} 


\section{The preparation of the couple to the Christian marriage: a pastoral-legal overview}

This brief analysis is inspired by the words of Pope Francis in his speech addressed to the officials of the Tribunal of the Roman Rota on 22nd January 2016: "a fortiori, pastoral urgency involving all Church structures is leading us toward a shared intention to provide adequate preparation for marriage in a kind of new catechumanate."

In light of the recent reform of the process for the declaration of nullity of marriage, we have studied in deep both the need for a link between the pastoral and judicial praxis - for the purpose of "proper preparation" of engaged couples for marriage - and the new needs that the reality of family demands.

So we have seen that from the court cases that the most frequent ones leading to the declaration of matrimonial nullity could be avoided and even forewarned by an effective preparation of engaged couples, with a true journey of rediscovery of the faith.

It is also clear from our research as the need for an ongoing tutoring for married couples in the first few years of marriage, is not only in conformity with the norms of the CIC, but for some time emerged in the documents of the Pastoral Family of the Holy See.

Parole-Chiave: Famiglia, matrimonio, catecumenato, Sinodo, tribunali, pastorale, Rota Romana.

KEY WORDS: Family, marriage, catechumenate, Synod, courts, pastoral, Roman Rota.

\section{Nota o Autorze:}

Prof. Antonio Interguglielmi - sacerdote della diocesi di Roma, professore di diritto canonico dello Studium Theologicum Galilaeae di Israele, affiliato alla Facoltà di Teologia della Pontificia Università Lateranense di Roma, ha pubblicato nel 2012 un libro nella Collana giuridica della LEV, $i$ Decreti singolari, dedicato all'attività amministrativa della Diocesi, e diversi articoli soprattutto di diritto canonico amministrativo e penale. E' Direttore dell'Ufficio per le Aggregazioni Laicali e le Confraternite della Diocesi di Roma e amministratore di diversi Enti ecclesiastici e Fondazioni della stessa Diocesi. 